\title{
Erratum
}

\section{Smiling reflects different emotions in men and women - ERRATUM}

\author{
Simine Vazire, Laura P. Naumann, Peter J. Rentfrow, and Samuel D. Gosling
}

doi:10.1017/S0140525X09991026, published by Cambridge University Press, 26 October 2009

On page 404 of the October 2009 issue of Behavioral and Brain Sciences, Figure 1 of the commentary by Vazire et al. on the target article by Vigil (2009) was duplicated and Figure 2 was omitted. The figures and their legends follow:

We regret the error.

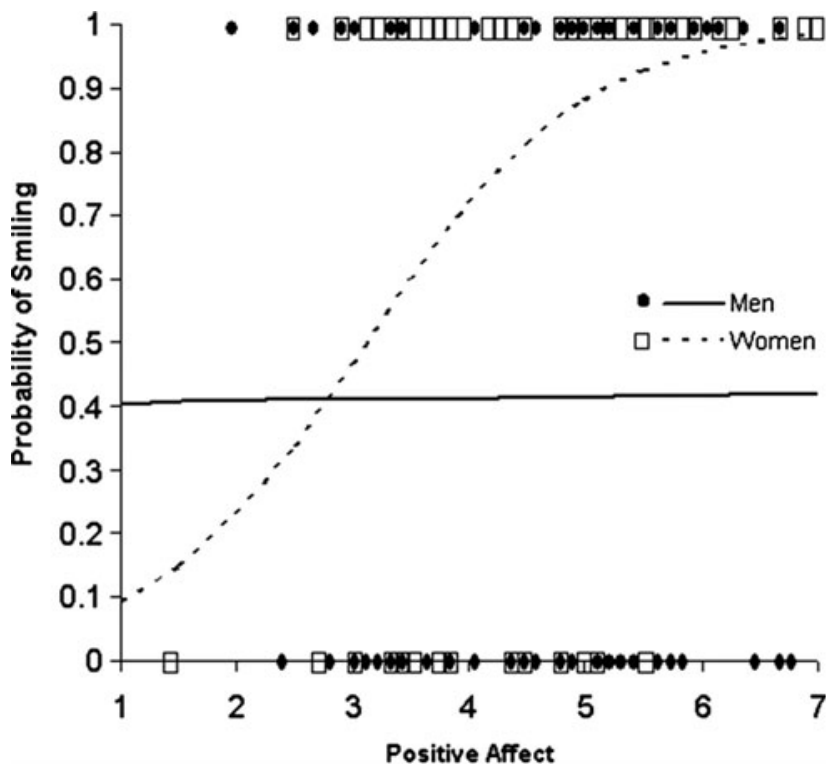

Figure 1. (Vazire et al.). Men's and women's probability of smiling as a function of positive affect. This plot is based on the results of two separate binary logistic regressions (one for men and one for women) predicting smiling from positive affect. The $\mathrm{x}$-axis represents the possible range of positive affect scores, and the $y$-axis represents probability of smiling based on the results of the regressions. The individual dots represent data from individual men (circles) and women (squares) who did and did not smile. Smiling was coded as a binary variable, so the dots appear on the $\mathrm{y}=0$ and $\mathrm{y}=1$ lines.

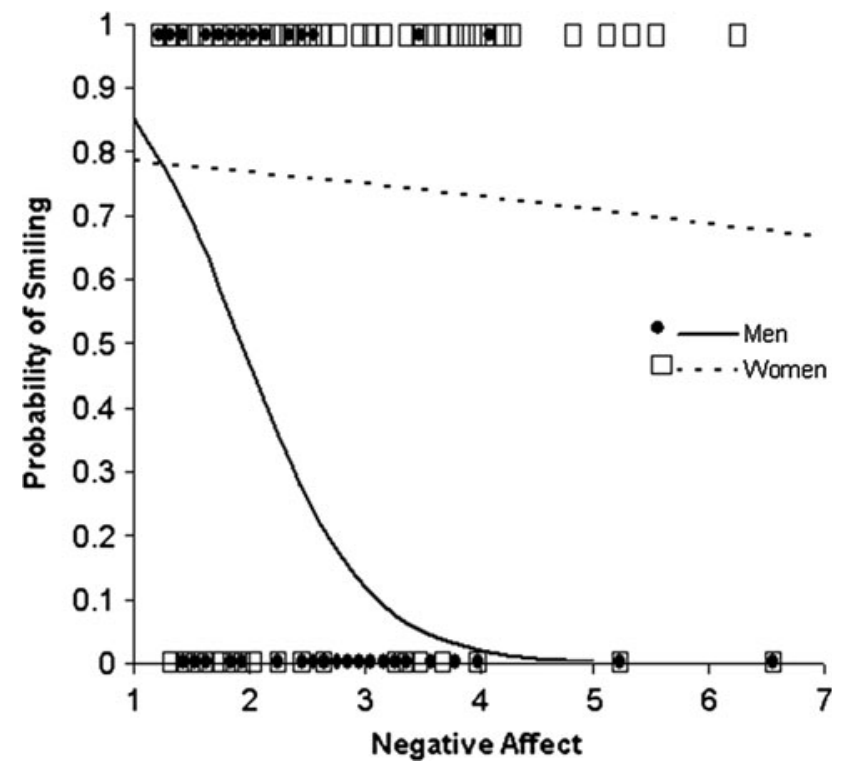

Figure 2. (Vazire et al.). Men's and women's probability of smiling as a function of negative affect. This plot is based on the results of two separate binary logistic regressions (one for men and one for women) predicting smiling from negative affect. The $\mathrm{x}$-axis represents the possible range of negative affect scores, and the y-axis represents probability of smiling based on the results of the regressions. The individual dots represent data from individual men (circles) and women (squares) who did and did not smile. Smiling was coded as a binary variable so the dots appear on the $\mathrm{y}=0$ and $\mathrm{y}=1$ lines.

\section{References}

Vazire, S., Naumann, L.P., Rentfrow, P.J. \& Gosling, S.D. (2009) Smiling reflects different emotions in men and women. Behavioral and Brain Sciences 32(5): 403-405. doi:10.1017/S0140525X09991026

Vigil, J.M. (2009) A socio-relational framework of sex differences in the expression of emotion. Behavioral and Brain Sciences 32(5):375-90. 